\title{
Epidemiology and Clinical Features of Coronavirus Disease 2019 in Moroccan Children
}

\author{
K El Fakiri, H Nassih, I Ait Sab, G Draiss And M Bouskraoui \\ From Department of Pediatrics, Child and Mother Hospital, Mohammed VI University Hospital Center, Marrakesh Medical and \\ Pharmacy Faculty, Caddy Ayad University, Marrakesh, Morocco.
}

Correspondence to: Dr Houda Nassih, Pediatric unit ' $B$ ', Department of Pediatrics, Child and Mother Hospital, Mohammed VI University Hospital Center, Marrakesh Medical and Pharmacy Faculty, Caddy Ayad University, City of Marrakesh, Morocco.houda.ped@gmail.com Received: May 14, 2020;

Initial review: June 19, 2020; Accepted: July 03, 2020
Objectives: This study aims to analyze the epidemiological and clinical features of coronavirus disease 19 (COVID-19) in a Moroccan pediatric population. Methods: A retrospective study of a cohort of 74 children with RT-PCR confirmed COVID-19. We collected information on clinical and laboratory features of all children (age $<18$ years) admitted between 2 March, 2020 and 1 April, 2020. Results: The mean (SD) age of the 74 children (40 girls) was 7 (1.5) years. The mean (SD) time from illness onset to diagnosis was 2 (1) days. 54 children were asymptomatic, while eight had fever, and five cases had cough. Recovery was after a mean (SD) of 12 (1) days. Conclusions: COVID-19 was mostly mild in the pediatric population in Morocco.

Keywords: Outcome, Pandemic, RT-PCR, SARS-CoV-2.

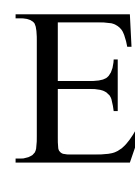
mergence of coronavirus disease 2019 (COVID-19) has attracted global attention, and the WHO has declared the severe acute respiratory syndrome coronavirus 2 (SARSCoV-2) infection as a pandemic. As of May 25, 2020, a total of 7756 cases, which $9 \%$ of them are children, occurred in Morocco [1]. Worldwide, it has been reported that the disease is mostly asymptomatic, or mild-moderate in nature in children [2-4]. To date, not much information is available about COVID-19 in Moroccan pediatric population. Our study aims to detail clinical features and outcome in Moroccan children infected with SARS-CoV-2 virus.

\section{METHODS}

We retrospectively reviewed records of 74 children confirmed with SARS-COV-2, who were managed in Mohammad VI university hospital of Marrakesh from March 2, 2020, to April 1, 2020. The ethics committee of our hospital approved this study. COVID-19 pediatric cases were defined as follows: Possible case: when a history of contact with a confirmed case of SARS-COV2 , and/or an acute respiratory infection of unknown etiology are present; and confirmation of infection was obtained from all patients at admission by detection of SARS-COV-2 nucleic acid on nasopharyngeal swab specimens using RT-PCR.
All confirmed COVID-19 cases $<18$ years were included except from newborns. Parents or guardians of all participants provided informed consent enrolled in this study. We collected data regarding epidemiological, demographical, clinical symptoms, laboratory measurements, imaging findings, management, and outcome. Laboratory workup carried out in all patients at diagnosis included complete blood count, CRP, procalcitonin, urea, creatinine, AST, ALT, PTT, aPTT, D-Dimer, ferritin, $\mathrm{LDH}, \mathrm{CPK}$, fibrinogen, and serum electrolytes. Computed tomography (CT) scan was done in those with severe manifestations. Patients were classified as follows: Asymptomatic infection (children without manifestations of clinical symptoms of COVID-19 testing positive to SARS-COV-2); Acute upper respiratory tract infection (children with fever, cough, pharyngeal pain, nasal congestion, fatigue, headache, myalgia or discomfort, and without signs of pneumonia by chest imaging or sepsis); Mild pneumonia (when children have a fever, respiratory symptoms such as cough, and chest imaging indicating pneumonia, but not reaching the criteria of severe pneumonia); Severe pneumonia (when any of the following criteria are present: $(i)$ increased respiratory rate: $\geq 60$ breaths $/ \min (<2$ years), $\geq 40$ breaths/min (between 2 and 5 years), $\geq 30$ breaths/min ( $\geq 5$ years); (ii) oxygen saturation $<94 \%$; (iii) hypoxia; (iv) disturbance of consciousness; and, ( $v$ ) food refusal or feeding difficulty, with signs of dehydration); 
and, Critical cases (who meet any of the following criteria and require ICU care: respiratory failure requiring mechanical ventilation, shock, or with other organ failure).

Treatment was prescribed according to the Moroccan Ministry of Health recommendations [5]. RT-PCR tests were done on the ninth, 14th, 21st, and 28th days from diagnosis. Recovery was declared when there was clinical improvement, child was afebrile for more than three days, and at least one negative RT-PCR result was obtained. All statistical data were processed using the Excel professional 2016 software.

\section{RESULTS}

Out of 74 children with confirmed COVID-19 included in our study, 34 (46\%) were boys. The median age was 7 years (range, 2 month - 17 year). Medical history was marked by two cases of mild intermittent asthma, one case of type 1 diabetes, one case of epilepsy, and one case of Down syndrome with intraventricular shunt. All the cases exhibited familial aggregation and had a history of close contact with their adult relatives who were diagnosed with COVID-19, except for one child who contacted the disease from a housemaid, and a second one who contacted from a neighbor. All our patients had satisfactory nutritional state, as well as normal growth parameters.

Fifty-four $(73 \%)$ children were asymptomatic. The remaining twenty patients had mild symptoms (Fig. 1), mainly symptoms of flu, with a mean (SD) time from illness onset to diagnoses of $2(0.5)$ days. Most frequent signs were fever $(10.8 \%)$, cough $(6.7 \%)$, rhinorrhea $(6.7 \%)$, and diarrhea (5.4\%). Hematological abnor-malities were marked by lymphocytosis in $8 \%$ of cases, while the rest were characterized by high creatinine phosphokinase (CPK), lactate dehydrogenase (LDH), and mild hepatic transaminitis $(<1.5$ times normal) (Table I).

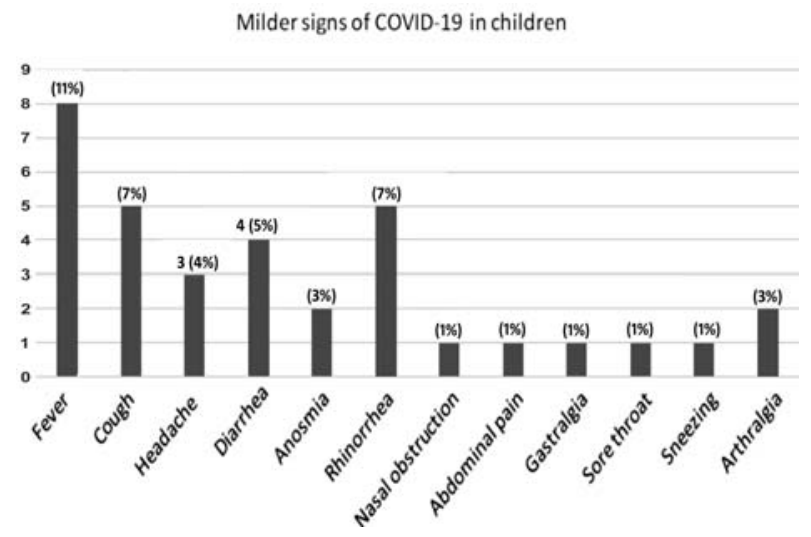

Fig. 1 Symptoms in children with confirmed COVID-19.
Management consisted of supportive care: hydration, antipyretics and nutritional support. The 15year-old girl was started on chloroquine $5 \mathrm{mg} / \mathrm{kg} / 12 \mathrm{~h}$ for five days (after documenting a normal electrocardiogram) with azithromycin $(10 \mathrm{mg} / \mathrm{kg}$ the first day; then $5 \mathrm{mg} / \mathrm{kg} /$ day for five days) along with vitamin $\mathrm{C}$ ( $1 \mathrm{~g}$ twice a day for ten days) and zinc ( $90 \mathrm{mg}$ twice a day for ten days). By the third day, evolution was marked by severe side effects (diplopia, nausea, epigastric pain), when we switched to hydroxychloroquine $(5 \mathrm{mg} / \mathrm{kg} / 12 \mathrm{~h}$ to complete a total of ten days). Subsequently, improvement was noted and no new side effects were seen. Breastfeeding was maintained in a two-months-old infant while respecting airborne and contact protection measures. No case was hospitalized in the intensive care unit or needed respiratory assistance. No patient died.

Resolution of symptoms occurred by the seventh day in most (80\%) cases. The average (SD) hospital stay was of 13 (3) days (range, 10-21 days). Negative RT-PCR results were obtained after a mean (SD) time of 14 (2) days ( $50 \%$ by ninth day, $75 \%$ by the 14 th day, $94 \%$ by the 21 st day), while four cases remained positive until the 28th day.

\section{DISCUSSION}

In Morocco, till date, approximately 600 children have been affected with COVID-19. Marrakesh is at the heart of the epidemic, with $19 \%$ of nationwide cases. All the pediatric cases are admitted to our center. Retrospective study of these cases found that our series agrees with those previously published, suggesting that most

Table I Laboratory Abnormalities in Pediatric Patients with Coronavirus Disease-2019 in Morocco $(N=74)$

\begin{tabular}{lc}
\hline Laboratory test & Number (\%) \\
\hline Complete blood count & \\
Anemia & $4(5)$ \\
Hyperleucocytosis & $2(3)$ \\
Leucopenia & $1(1)$ \\
Lymphopenia & $2(3)$ \\
Lymphocytosis & $6(8)$ \\
AST $(\geq 40$ IU/L) & $22(30)$ \\
ALT $(\geq 40$ IU/L) & $22(30)$ \\
Ferritin $(>150 \mathrm{ng} / \mathrm{mL})$ & $2(3)$ \\
LDH $(>290 \mathrm{IU} / \mathrm{L})$ & $32(43)$ \\
CPK $(>25 \mathrm{IU} / \mathrm{L})$ & $10(13.5)$ \\
Procalcitonin $(>0.5 \mathrm{ng} / \mathrm{mL})$ & $9(12)$ \\
\hline
\end{tabular}

AST - Aspartate aminotransferase; ALT - alanine aminotransferase; LDH - lactic dehydrogenase; CPK - creatinine phosphokinase. 


\section{WHAT THIS STUDY ADDS?}

- Our study describes the North African experience with COVID-19 in children.

pediatric COVID-19 cases are females [6]. Children of all ages were susceptible to COVID-19 similar to other studies $[7,8]$. The most affected proportion of children were aged between 10 and 14 years, with a mean age similar to a Korean report [9], while a Spanish series found that most affected children were younger [6]. A Chinese series reported that over $90 \%$ of children were either asymptomatic or with mild-moderate manifestations [4]. The majority of our cases were asymptomatic because these infections were recognized mostly through contact tracing. Median time from illness onset to diagnosis was similar to the Chinese series [4]. All the symptomatic cases were upper respiratory infections, while other series found more severe cases [9-11]. Lymphopenia has been reported in COVID-19 previously also [9], and was seen in $3 \%$ of our children.

The Moroccan ministry of health approved in early April, 2020, a nationwide protocol using chloroquine or hydroxychloroquine associated with azithromycin in all COVID-19 severe pediatric cases [5]. We used this protocol in only one case with an immunocompromized condition (type 1 diabetes). Finally, we were well aware of the impact of the psychological state on the well-being of our children, so as recommended by Massimo, et al., [12], we organized various activities such as anniversary parties, as well as providing hospitalized children with toys and books to alleviate loneliness.

In conclusion, COVID-19 in Moroccan children seems to be mild, with non-specific clinical and biological findings, and with a rare need of specific treatment. Other studies are necessary to verify these findings from this preliminary report.

Ethics Clearance: Ethics Committee of Mohammed VI University Hospital Center of Marrakesh, Morocco; No. SD269815/20 dated May 02, 2020.

Contributors: KF: concept and designed the study, analyzed data and drafted the manuscript; HN: helped in data analysis and writing; GD, MB, IS: supervised data analysis. All authors approved the final manuscript.

Funding: None; Competing interest: None stated.

\section{REFERENCES}

1. The Official Coronavirus Portal in Morocco [Internet]. Available from: http://www.covidmaroc.ma/pages/
Accueil.aspx. Accessed May 12, 2020.

2. Wu Z, McGoogan JM. Characteristics of and important lessons from the coronavirus disease 2019 (COVID-19) outbreak in China: Summary of a report of 72314 cases from the Chinese Center for Disease Control and Prevention. JAMA. 2020;323:1239-42.

3. Balasubramanian S, Rao NM, Goenka A, Roderick M, Ramanan AV. Coronavirus disease 2019 (COVID-19) in children - What we know so far and what we do not. Indian Pediatr. 2020;57:435-42.

4. Dong Y, Mo X, Hu Y, Qi X, Jiang F, Jiang Z, et al. Epidemiology of COVID-19 among children in China. Pediatrics. 2020; e20200702.

5. Moroccan Ministry of health recommendations for the therapeutic management of confirmed COVID-19 cases [Internet]. Available from: http://www.covidmaroc.ma/ Documents/2020/coronavirus/PS/Covid-19Prise $\% 20$ en $\%$ 20charge $\% 20$ th $\%$ C3\%A9rapeutique\%20des $\% 20$ cas $\% 20$ confirm\%C3\%A9s\%20(23mars2020).pdf. Accessed May 23, 2020.

6. Tagarro A, Epalza C, Santos M, Sanz-Santaeufemia FJ, Otheo E, Moraleda C, et al. Screening and severity of coronavirus disease 2019 (COVID-19) in children in Madrid, Spain. JAMA Pediatr [Internet]. Available from: https://jamanetwork.com/journals/jamapediatrics/full article/276439. Accessed May 27, 2020.

7. Wei Xia, Jianbo Shao, Yu Guo, Xuehua Peng, Zhen Li, Daoyu $\mathrm{Hu}$. Clinical and CT features in pediatric patients with COVID 19 infection: Different points from adults. Pediatr Pulmonol. 2020;55:1169-74.

8. Choi S-H, Kim HW, Kang J-M, Kim DH, Cho EY. Epidemiology and clinical features of coronavirus disease 2019 in children. Clin Exp Pediatr. 2020;63:125-32.

9. Liu W, Zhang Q, Chen J, Xiang R, Song H, Shu S, et al. Detection of COVID-19 in children in early January 2020 in Wuhan, China. N Engl Med. 2020;382:1370-1.

10. Wang D, Ju XL, Xie F, Lu Y, Li FY, Huang HH, et al. Clinical analysis of 31 cases of 2019 novel coronavirus infection in children from six provinces (autonomous region) of northern China. Zhonghua $\mathrm{Er} \mathrm{Ke} \mathrm{Za} \mathrm{Zhi.}$ 2020;58:E011.

11. Feng K, Yun YX, Wang XF, Yang GD, Zheng YJ, Lin CM, et al. Analysis of CT features of 15 children with 2019 novel coronavirus infection. Zhonghua $\mathrm{Er} \mathrm{Ke} \mathrm{Za} Z \mathrm{Zhi}$. 2020;58:275-8.

12. Pettoello-Mantovani M, Pop TL, Mestrovic J, Ferrara P, Giardino I, Carrasco-Sanz A, et al. Fostering resilience in children: The essential role of healthcare professionals and families. J Pediatr. 2019;205:298-99.e1. 